\title{
New results on the Stackelberg-Nash exact control of linear parabolic equations
}

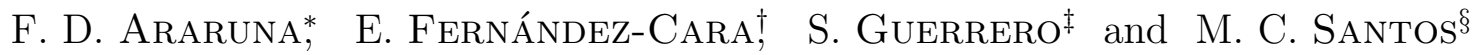

\begin{abstract}
This paper is concerned with Stackelberg-Nash strategies to control parabolic equations. We have one control, the leader, that is responsible for a null controllability property; additionally, we have a couple of controls, called the followers, that provides a Nash equilibrium for two cost functionals. This is a classical situation in many fields of science and, in mathematics, leads to a lot of interesting questions and open problems and possesses many applications. In the main result, we prove the existence of a leader such that the corresponding controlled system is driven to zero. This way, we improve some questions that were left open in previous works.
\end{abstract}

\section{Introduction}

There are plenty of situations where several controls are required in order to drive a system to one or more objectives. Usually, if we assign different roles to the controls, we speak of hierarchic control. In the case of a system governed by a PDE, this concept was introduced by J.-L. Lions (see [15, 16], where some techniques are presented). These works motivated the study of the subject and a lot of other results appeared; see for instance $[4,5,12,19,20]$.

All these previous works combine the multicriteria optimization concepts and arguments and approximate controllability. In the context of null controllability, few is known; see [1] for some first results.

In this paper, we solve a question that was left open in [1]. The solution requires some careful computations based on new Carleman estimates. Let us be more precise.

Let $\Omega \subset \mathbb{R}^{n}$ be a bounded domain whose boundary $\Gamma$ is regular enough. Let $T>0$ be given and define $Q:=\Omega \times(0, T)$, with lateral boundary $\Sigma:=\partial \Omega \times(0, T)$. In the sequel, we will denote by $C$ a generic positive constant which may differ from line to line. Sometimes, we will write $C(\Omega), C(\Omega, T)$, etc. to indicate the data on which $C$ depends. The usual norm and scalar product in $L^{2}(\Omega)$ will be respectively denoted by $\|\cdot\|$ and $(\cdot, \cdot)$.

Let us consider the linear system

$$
\begin{cases}y_{t}-\Delta y+a(x, t) y=f 1_{\mathcal{O}}+v^{1} 1_{\mathcal{O}_{1}}+v^{2} 1_{\mathcal{O}_{2}} & \text { in } Q, \\ y=0 & \text { on } \Sigma, \\ y(\cdot, 0)=y^{0} & \text { in } \Omega,\end{cases}
$$

where $y=y(x, t)$ is the state, $a \in L^{\infty}(Q)$ and $y^{0}=y^{0}(x)$ is prescribed. In (1), the set $\mathcal{O} \subset \Omega$ is the main control domain and $\mathcal{O}_{1}, \mathcal{O}_{2} \subset \Omega$ are the secondary control domains (all of them are supposed to be small); $1_{\mathcal{O}}, 1_{\mathcal{O}_{1}}$ and $1_{\mathcal{O}_{2}}$ are the characteristic functions of $\mathcal{O}, \mathcal{O}_{1}$ and $\mathcal{O}_{2}$, respectively; the controls are the leader $f=f(x, t)$ and the followers $v^{1}=v^{1}(x, t)$ and $v^{2}=v^{2}(x, t)$.

\footnotetext{
*Dpto. de Matemática, Universidade Federal da Paraíba, 58051-900, João Pessoa - PB, Brazil. E-mail: fagner@mat.ufpb.br. Partially supported by INCTMat, CAPES, CNPq (Brazil) and MathAmSud COSIP.

${ }^{\dagger}$ Dpto. EDAN and IMUS, University of Sevilla, Aptdo. 1160, 41080 Sevilla, Spain (cara@us.es). Partially supported by grant MTM2010-15592, DGI-MICINN (Spain) and CAPES (Brazil).

${ }^{\ddagger}$ Laboratoire Jacques-Louis Lions, Université Pierre et Marie Curie, 75252 Paris Cédex 05, France. E-mail: guerrero@ann.jussieu.fr. Partially supported by MathAmSud COSIP.

$\S$ Dpto. de Matemática, Universidade Federal da Pernambuco, 50670-901, Recife - PE, Brazil. E-mail: mauricio@dmat.ufpe.br. Partially supported by CAPES (Brazil) and MathAmSud COSIP.
} 
Let $\mathcal{O}_{1, d}, \mathcal{O}_{2, d} \subset \Omega$ be open sets, representing observation domains for the followers. We will consider the (secondary) functionals

$$
J_{i}\left(f ; v^{1}, v^{2}\right):=\frac{\alpha_{i}}{2} \iint_{\mathcal{O}_{i, d} \times(0, T)}\left|y-y_{i, d}\right|^{2} d x d t+\frac{\mu}{2} \iint_{\mathcal{O}_{i} \times(0, T)}\left|v^{i}\right|^{2} d x d t, \quad i=1,2,
$$

and the main functional

$$
J(f):=\frac{1}{2} \iint_{\mathcal{O} \times(0, T)}|f|^{2} d x d t
$$

where the $\alpha_{i}>0$ and $\mu>0$ are constants and the $y_{i, d}=y_{i, d}(x, t)$ are given functions.

The structure of the control process can be described as follows:

1. For each leader $f$, the followers $v^{1}$ and $v^{2}$ intend to be a Nash equilibrium for the $\operatorname{costs} J_{i}(i=1,2)$.

In other words, once $f$ has been fixed, we look for a couple $\left(v^{1}, v^{2}\right)$ with $v^{i} \in L^{2}\left(\mathcal{O}_{i} \times(0, T)\right)$ such that

$$
J_{1}\left(f ; v^{1}, v^{2}\right)=\min _{\hat{v}^{1}} J_{1}\left(f ; \hat{v}^{1}, v^{2}\right), \quad J_{2}\left(f ; v^{1}, v^{2}\right)=\min _{\hat{v}^{2}} J_{2}\left(f ; v^{1}, \hat{v}^{2}\right) .
$$

Note that, if the functionals $J_{i}(i=1,2)$ are $C^{1}$ and convex, then $\left(v^{1}, v^{2}\right)$ is a Nash equilibrium if and only if

$$
J_{1}^{\prime}\left(f ; v^{1}, v^{2}\right)\left(\hat{v}^{1}, 0\right)=0, \quad \forall \hat{v}^{1} \in L^{2}\left(\mathcal{O}_{1} \times(0, T)\right), \quad v^{i} \in L^{2}\left(\mathcal{O}_{i} \times(0, T)\right)
$$

and

$$
J_{2}^{\prime}\left(f ; v^{1}, v^{2}\right)\left(0, \hat{v}^{2}\right)=0, \quad \forall \hat{v}^{2} \in L^{2}\left(\mathcal{O}_{2} \times(0, T)\right), \quad v^{i} \in L^{2}\left(\mathcal{O}_{i} \times(0, T)\right) .
$$

(In fact, this is also true if $J_{i}$ is $C^{1}$ and convex in the $i$-th variable.)

2. Let us fix an uncontrolled trajectory of (1), that is, a sufficiently regular solution to the system

$$
\begin{cases}\bar{y}_{t}-\Delta \bar{y}+a(x, t) \bar{y}=0 & \text { in } \quad Q, \\ \bar{y}=0 & \text { on } \Sigma, \\ \bar{y}(\cdot, 0)=\bar{y}^{0} & \text { in } \Omega .\end{cases}
$$

Once the Nash equilibrium has been identified and fixed for each $f$, we look for an optimal control $\hat{f} \in L^{2}(\mathcal{O} \times(0, T))$ such that

$$
J(\hat{f})=\min _{f} J(f),
$$

subject to the exact controllability restriction

$$
y(\cdot, T)=\bar{y}(\cdot, T) \quad \text { in } \quad \Omega .
$$

In [1] it is proved that, if $\mu$ is large enough, for every $f \in L^{2}(\mathcal{O} \times(0, T))$ there exists a unique Nash equilibrium $\left(v^{1}, v^{2}\right)$ for $\left(J_{1}, J_{2}\right)$, given by

$$
v_{i}=-\frac{1}{\mu} \phi^{i} 1_{\mathcal{O}_{i}}, \quad i=1,2,
$$

where $\left(y, \phi^{1}, \phi^{2}\right)$ is the unique solution to the optimality system

$$
\begin{cases}y_{t}-\Delta y+a(x, t) y=f 1_{\mathcal{O}}-\sum_{i=1}^{2} \frac{1}{\mu} \phi^{i} 1_{\mathcal{O}_{i}} & \text { in } \quad Q, \\ -\phi_{t}^{i}-\Delta \phi^{i}+a(x, t) \phi^{i}=\alpha_{i}\left(y-y_{i, d}\right) 1_{\mathcal{O}_{i, d}} & \text { in } \quad Q, \\ y=0, \quad \phi^{i}=0 & \text { on } \Sigma, \\ y(\cdot, 0)=y^{0}, \quad \phi^{i}(\cdot, T)=0 & \text { in } \quad \Omega .\end{cases}
$$

The main result of this paper concerns the exact controllability to the trajectories of (1)-(2). It is the following: 
Theorem 1. Suppose that

$$
\mathcal{O}_{i, d} \cap \mathcal{O} \neq \emptyset, \quad i=1,2 .
$$

Also, assume that one of the following two conditions holds:

$$
\mathcal{O}_{1, d}=\mathcal{O}_{2, d}
$$

or

$$
\mathcal{O}_{1, d} \cap \mathcal{O} \neq \mathcal{O}_{2, d} \cap \mathcal{O} \text {. }
$$

Then, there exists $\mu_{0}>0$, only depending on $\Omega, \mathcal{O}, T, \mathcal{O}_{i}, \mathcal{O}_{i, d}, \alpha_{i}$ and $\|a\|_{L^{\infty}(Q)}$ and a positive function $\hat{\rho}=\hat{\rho}(t)$ blowing up at $t=T$ such that, if $\mu \geq \mu_{0}$, the $y_{i, d}$ are such that

$$
\iint_{\mathcal{O}_{i, d} \times(0, T)} \hat{\rho}^{2}\left|\bar{y}-y_{i, d}\right|^{2} d x d t<+\infty, \quad i=1,2
$$

and $\bar{y}$ is the unique solution to (3) associated to the initial state $\bar{y}^{0} \in L^{2}(\Omega)$, there exist controls $f \in$ $L^{2}(\mathcal{O} \times(0, T))$ and associated Nash equilibria $\left(v^{1}, v^{2}\right)$ such that the corresponding solutions to (1) satisfy (4).

Remark 2. It is worth mentioning that, in [1], the authors have proved this result in the particular case in which (6) and (7) are satisfied. Fig. 1-3 illustrate some situations where this fails and (6) and (8) hold simultaneously.

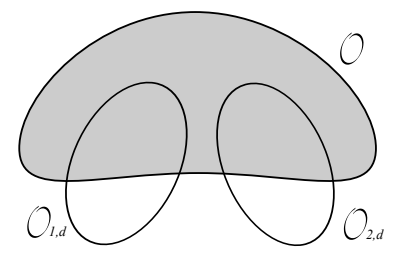

Figure 1

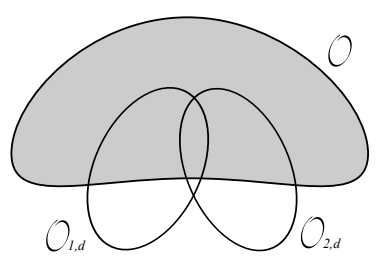

Figure 2

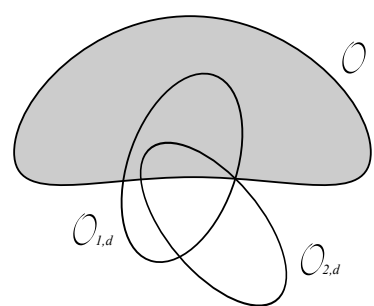

Figure 3

Note that, if we introduce the new variable $z=y-\bar{y},(5)$ can be rewritten in the form

$$
\begin{cases}z_{t}-\Delta z+a(x, t) z=f 1_{\mathcal{O}}-\sum_{i=1}^{2} \frac{1}{\mu} \phi^{i} 1_{\mathcal{O}_{i}} & \text { in } \quad Q, \\ -\phi_{t}^{i}-\Delta \phi^{i}+a(x, t) \phi^{i}=\alpha_{i}\left(z-z_{i, d}\right) 1_{\mathcal{O}_{i, d}} & \text { in } \quad Q, \\ z=0, \quad \phi^{i}=0 & \text { on } \Sigma, \\ z(\cdot, 0)=z^{0}, \quad \phi^{i}(\cdot, T)=0 & \text { in } \quad \Omega,\end{cases}
$$

where $z_{i, d}=y_{i, d}-\bar{y}$ and $z^{0}=y^{0}-\bar{y}^{0}$ and (4) is equivalent to the null controllability property for $z$, that is,

$$
z(\cdot, T)=0 \quad \text { in } \quad \Omega .
$$

The proof of Theorem 1 relies on some duality arguments which reduce the null controllability property of a linear system to an observability inequality for the solutions to the associated adjoint system. In our case, the adjoint of (9) is

$$
\begin{cases}-\psi_{t}-\Delta \psi+a(x, t) \psi=\sum_{i=1}^{2} \alpha_{i} \gamma^{i} 1_{\mathcal{O}_{i, d}} & \text { in } \quad Q, \\ \gamma_{t}^{i}-\Delta \gamma^{i}+a(x, t) \gamma^{i}=-\frac{1}{\mu} \psi 1_{\mathcal{O}_{i}} & \text { in } \quad Q, \\ \psi=0, \quad \gamma^{i}=0 & \text { on } \Sigma, \\ \psi(\cdot, T)=\psi^{T}, \quad \gamma^{i}(\cdot, 0)=0 & \text { in } \Omega .\end{cases}
$$


For the proof of the observability of (11), as usual, we must use Carleman estimates. Thus, the task is to estimate globally all the variables $\psi, \gamma^{1}$ and $\gamma^{2}$ by just one observation $(\psi$ in $\mathcal{O} \times(0, T))$. This is not trivial, especially when $\mathcal{O}_{1, d} \neq \mathcal{O}_{2, d}$. In fact, in [1], the assumption $\mathcal{O}_{1, d}=\mathcal{O}_{2, d}$ was needed.

The main idea in this paper is to use not one Imanuvilov function, but two. This is possible thanks to Lemma 5 (see below, in Section 2). Actually, we believe that this lemma, used together with the arguments in the proof of Proposition 11, can be a useful tool for the solution of other control problems. For instance, to control a $3 \times 3$ system with one scalar control.

The observability estimate for (11) is given in the following result:

Theorem 3. Under the assumptions of Theorem 1, there exists a constant $C>0$, depending only on $\Omega, \mathcal{O}$, $T, \mathcal{O}_{i}, \mathcal{O}_{i, d}, \alpha_{i}$ and $\|a\|_{L^{\infty}(Q)}$ and a weight $\hat{\rho}=\hat{\rho}(t)$ such that

$$
\int_{\Omega}|\psi(x, 0)|^{2} d x+\sum_{i=1}^{2} \iint_{Q} \hat{\rho}^{-2}\left|\gamma^{i}\right|^{2} d x d t \leqslant C \iint_{\mathcal{O} \times(0, T)}|\psi|^{2} d x d t
$$

In order to prove (12), we will use some suitable Carleman estimates with a specific and unusual choice of the weight functions. This will be done precisely in the following section.

Remark 4. The arguments in Section 3 of [1] concerning semilinear problems can be adapted to the present setting. This allows to prove a result similar to Theorem 1 for the system

$$
\begin{cases}y_{t}-\Delta y+a(x, t) y=F(y)+f 1_{\mathcal{O}}+v^{1} 1_{\mathcal{O}_{1}}+v^{2} 1_{\mathcal{O}_{2}} & \text { in } Q, \\ y=0 & \text { on } \Sigma, \\ y(\cdot, 0)=y^{0} & \text { in } \Omega,\end{cases}
$$

where (for instance) $F \in W^{1, \infty}(\mathbb{R})$. As there, we have to introduce the concept of Nash quasi-equilibrium and then an appropriate fixed-point mapping. We omit the details, since the process is rather standard and well known.

The paper is organized as follows. In Section 2, we introduce the weight functions needed to prove some Carleman estimates. In Section 3, we prove these Carleman estimates. In Section 4, we deduce the observability inequality (12) and, also, that (12) implies (10).

\section{Some previous results}

In this section we define the weight functions needed in the proof of Theorem 1. We will also recall some known Carleman inequalities.

Let us introduce a nonempty open set $\widetilde{\mathcal{O}} \subset \subset \mathcal{O}$ such that $\mathcal{O}_{i, d} \cap \widetilde{\mathcal{O}} \neq \emptyset$ for $i=1,2$ and the nonempty connected open sets $\omega_{i}$ with

$$
\omega_{i} \subset \subset \mathcal{O}_{i, d} \cap \widetilde{\mathcal{O}}, \quad i=1,2, \quad \omega_{1} \cap \omega_{2}=\emptyset .
$$

Observe that this is possible thanks to assumptions (6) and (8).

The next result will be crucial:

Lemma 5. There exist functions $\eta_{i} \in C^{2}(\bar{\Omega})(i=1,2)$ such that

$$
\left\{\begin{array}{l}
\eta_{i}>0 \quad \text { in } \Omega, \quad \eta_{i}=0 \quad \text { on } \partial \Omega, \\
\left|\nabla \eta_{i}\right|>0 \quad \text { in } \quad \bar{\Omega} \backslash \omega_{i}, \quad \eta_{1}=\eta_{2} \quad \text { in } \Omega \backslash \widetilde{O} .
\end{array}\right.
$$

The proof of Lemma 5 can be found in an Appendix (Section 5), at the end of the paper.

Remark 6. We know from [8, Lemma 1.1] that, for any open set $\omega_{0} \subset \Omega$, there exists $\eta_{0} \in C^{2}(\bar{\Omega})$ satisfying

$$
\left\{\begin{array}{l}
\eta_{0}>0 \text { in } \Omega, \quad \eta_{0}=0 \text { on } \partial \Omega, \\
\left|\nabla \eta_{0}\right|>0 \text { in } \bar{\Omega} \backslash \omega_{0} .
\end{array}\right.
$$

From the proof of this lemma (see [8, pp. 20-21]), it is also clear that the function $\eta_{0}$ can be chosen with a finite number of critical points. 
Remark 7. Lemma 5 establishes the existence of functions $\eta_{1}$ and $\eta_{2}$ which coincide outside $\widetilde{\mathcal{O}}$ but may be very different inside $\widetilde{\mathcal{O}}$. Nevertheless, it will be seen in the proof that one can find $\eta_{1}$ and $\eta_{2}$ satisfying $\left\|\eta_{1}\right\|_{\infty}=\left\|\eta_{2}\right\|_{\infty}$.

Remark 8. From (6), (8) and (14), we see that it can be assumed that either

$$
\omega_{1} \cap \mathcal{O}_{2, d}=\emptyset \quad \text { and } \quad \omega_{2} \cap \mathcal{O}_{1, d}=\emptyset
$$

or

$$
\omega_{i} \subset \mathcal{O}_{j, d} \text { and } \omega_{j} \cap \mathcal{O}_{i, d}=\emptyset \text {, with }(i, j)=(1,2) \text { or }(i, j)=(2,1) .
$$

The cases (15) and (16) (with $(i, j)=(2,1)$ ) correspond, respectively, to Fig. 1 and Fig. 3.

Let us introduce the weight functions

$$
\sigma_{i}(x, t):=\frac{e^{4 \lambda\left\|\eta_{i}\right\|_{\infty}}-e^{\lambda\left(2\left\|\eta_{i}\right\|_{\infty}+\eta_{i}(x)\right)}}{t(T-t)}, \quad \xi_{i}(x, t):=\frac{e^{\lambda\left(2\left\|\eta_{i}\right\|_{\infty}+\eta_{i}(x)\right)}}{t(T-t)}
$$

and the notation

$$
I_{m}^{i}(\psi):=s^{m-4} \lambda^{m-3} \iint_{Q} e^{-2 s \sigma_{i}}\left(\xi_{i}\right)^{m-4}\left(\left|\psi_{t}\right|^{2}+|\Delta \psi|^{2}\right) d x d t+L_{m}^{i}(\psi),
$$

where

$$
\begin{aligned}
L_{m}^{i}(\psi): & =s^{m-2} \lambda^{m-1} \iint_{Q} e^{-2 s \sigma_{i}}\left(\xi_{i}\right)^{m-2}|\nabla \psi|^{2} d x d t \\
& +s^{m} \lambda^{m+1} \iint_{Q} e^{-2 s \sigma_{i}}\left(\xi_{i}\right)^{m}|\psi|^{2} d x d t .
\end{aligned}
$$

We will need some known results concerning Carleman estimates for parabolic PDEs. Thus, let us consider the system

$$
\begin{cases}-u_{t}-\Delta u+a u=f+\sum_{k=1}^{n} \partial_{k} f_{k} & \text { in } Q, \\ u=0 & \text { on } \Sigma, \\ u(\cdot, T)=u^{T} & \text { in } \Omega,\end{cases}
$$

where $u^{T} \in L^{2}(\Omega)$ and $f, f_{1}, \ldots, f_{n} \in L^{2}(Q)$.

We have the following:

Proposition 9. Assume that, in (17), $f_{k}=0$ for all $k=1, \ldots, n$. Then, there exists $C(\Omega, \mathcal{O})>0$ such that, for every $s \geq C\left(T+T^{2}\right)$ and every $\lambda \geq C$, the solution $u$ to (17) associated to $u^{T} \in L^{2}(\Omega)$ and $f \in L^{2}(Q)$ satisfies

$$
I_{m}^{j}(u) \leqslant C\left(s^{m} \lambda^{m+1} \iint_{\omega_{j} \times(0, T)} e^{-2 s \sigma_{j}}\left(\xi_{j}\right)^{m}|u|^{2} d x d t+s^{m-3} \lambda^{m-3} \iint_{Q} e^{-2 s \sigma_{j}}\left(\xi_{j}\right)^{m-3}|f|^{2} d x d t\right), j=1,2 .
$$

If the functions $f_{k}$ are not necessarily zero, the following holds:

Proposition 10. There exists $C(\Omega, \mathcal{O})>0$ such that, for every $s \geq C\left(T+T^{2}\right)$ and every $\lambda \geq C$, the solution $u$ to (17) associated to $u^{T} \in L^{2}(\Omega), f \in L^{2}(Q)$ and the $f_{k} \in L^{2}(Q)$ satisfies

$$
\begin{aligned}
L_{m}^{j}(u) \leqslant & C\left(s^{m} \lambda^{m+1} \iint_{\omega_{j} \times(0, T)} e^{-2 s \sigma_{j}}\left(\xi_{j}\right)^{m}|u|^{2} d x d t+s^{m-3} \lambda^{m-3} \iint_{Q} e^{-2 s \sigma_{j}}\left(\xi_{j}\right)^{m-3}|f|^{2} d x d t\right. \\
& \left.+s^{m-1} \lambda^{m-1} \sum_{k=1}^{2} \iint_{Q} e^{-2 s \sigma_{j}}\left(\xi_{j}\right)^{m-1}\left|f_{k}\right|^{2} d x d t\right), \quad j=1,2 .
\end{aligned}
$$

These results are nowadays well known. For instance, when $m=3$, their proofs can be respectively found in $[8]$ and $[14]$. 


\section{A new Carleman inequality}

In this section, we will prove a suitable Carleman inequality for the solutions to the adjoint system (11). In [1], the authors proved a similar estimate assuming that (6) and (7) hold.

Proposition 11. Assume that (6) and (8) are satisfied. Then, there exists $C(\Omega, \mathcal{O})>0$ such that, for every $s \geq C\left(T+T^{2}\right)$ and every $\lambda \geq C$, the solution $\left(\psi, \gamma^{1}, \gamma^{2}\right)$ to (11) associated to $\psi^{T} \in L^{2}(\Omega)$ satisfies the following:

(i) If (15) holds, then

$$
\begin{aligned}
I_{0}^{1}\left(\gamma^{1}\right) & +I_{0}^{2}\left(\gamma^{2}\right)+s^{-3} \lambda^{-2} \iint_{Q} e^{-2 s \sigma_{1}}\left(\xi_{1}\right)^{-3}|\psi|^{2} d x d t \\
& \leqslant C s^{4} \lambda^{5} \iint_{\mathcal{O} \times(0, T)}\left(e^{-2 s \sigma_{1}}\left(\xi_{1}\right)^{4}+e^{-2 s \sigma_{2}}\left(\xi_{2}\right)^{4}\right)|\psi|^{2} d x d t .
\end{aligned}
$$

(ii) If (16) holds for $(i, j)=\left(i_{0}, j_{0}\right)$, with $\left(i_{0}, j_{0}\right)=(1,2)$ or $\left(i_{0}, j_{0}\right)=(2,1)$, then

$$
\begin{aligned}
& I_{0}^{j_{0}}\left(\gamma^{j_{0}}\right)+I_{0}^{i_{0}}(h)+s^{-3} \lambda^{-2} \iint_{Q} e^{-2 s \sigma_{j_{0}}}\left(\xi_{j_{0}}\right)^{-3}|\psi|^{2} d x d t \\
& \leqslant C s^{4} \lambda^{5} \iint_{\mathcal{O} \times(0, T)}\left(e^{-2 s \sigma_{1}}\left(\xi_{1}\right)^{4}+e^{-2 s \sigma_{2}}\left(\xi_{2}\right)^{4}\right)|\psi|^{2} d x d t,
\end{aligned}
$$

where we have denoted $h:=\alpha_{1} \gamma_{1}+\alpha_{2} \gamma_{2}$.

Remark 12. In order to prove the above Carleman inequalities, we will have to work with the equation satisfied by $\gamma^{i}$. However, the terms involving $\gamma^{i}$ and $h$ in the left-hand side of (18) and (19) will not be needed later, in the proof of the observability inequality (12).

Proof. The proofs of (i) and (ii) are slightly different and will be presented separately.

- Proof of (i). We first apply Proposition 9 for $m=0$ and $j=i$ to the functions $(x, t) \mapsto \gamma^{i}(x, T-t)$ for $i=1,2$. This gives

$$
I_{0}^{i}\left(\gamma^{i}\right) \leqslant C\left(\lambda \iint_{\omega_{i} \times(0, T)} e^{-2 s \sigma_{i}}\left|\gamma^{i}\right|^{2} d x d t+s^{-3} \lambda^{-3} \iint_{\mathcal{O}_{i} \times(0, T)} e^{-2 s \sigma_{i}}\left(\xi_{i}\right)^{-3}|\psi|^{2} d x d t\right) .
$$

Now, let $\theta_{3} \in C^{2}(\bar{\Omega})$ be such that

$$
\begin{cases}\theta_{3}(x)=0 & \text { for } x \in \widetilde{\mathcal{O}} \\ \theta_{3}(x)=1 & \text { for } x \in \Omega \backslash \overline{\mathcal{O}}\end{cases}
$$

From (11), we find that

$$
\begin{cases}-\left(\theta_{3} \psi\right)_{t}-\Delta\left(\theta_{3} \psi\right)+a\left(\theta_{3} \psi\right)=\sum_{j=1}^{2} \alpha_{j} \theta_{3} \gamma^{j} 1_{\mathcal{O}_{j, d}}-2 \nabla \cdot\left(\psi \nabla \theta_{3}\right)+\Delta \theta_{3} \psi & \text { in } Q, \\ \theta_{3} \psi=0 & \text { on } \Sigma, \\ \left(\theta_{3} \psi\right)(\cdot, T)=\theta_{3} \psi^{T} & \text { in } \Omega .\end{cases}
$$


Then, we apply Proposition 10 for $m=-3$ and $j=1$ to $\theta_{3} \psi$ and we get

$$
\begin{aligned}
& L_{-3}^{1}\left(\theta_{3} \psi\right) \leqslant C\left(s^{-3} \lambda^{-2} \iint_{\omega_{1} \times(0, T)} e^{-2 s \sigma_{1}}\left(\xi_{1}\right)^{-3}|\psi|^{2} d x d t\right. \\
& \quad+s^{-6} \lambda^{-6} \iint_{\mathcal{O}_{1, d} \times(0, T)} e^{-2 s \sigma_{1}}\left(\xi_{1}\right)^{-6}\left|\gamma^{1}\right|^{2} d x d t+s^{-6} \lambda^{-6} \iint_{\mathcal{O}_{2, d} \times(0, T)} e^{-2 s \sigma_{1}}\left(\xi_{1}\right)^{-6}\left|\theta_{3} \gamma^{2}\right|^{2} d x d t \\
& \left.\quad+s^{-4} \lambda^{-4} \iint_{\mathcal{O} \times(0, T)} e^{-2 s \sigma_{1}}\left(\xi_{1}\right)^{-4}|\psi|^{2} d x d t\right) .
\end{aligned}
$$

Using the fact that $\theta_{3}$ equals 1 in $\Omega \backslash \overline{\mathcal{O}}$, we deduce that

$$
L_{-3}^{1}\left(\theta_{3} \psi\right) \geq s^{-3} \lambda^{-2} \iint_{Q} e^{-2 s \sigma_{1}}\left(\xi_{1}\right)^{-3}|\psi|^{2} d x d t-s^{-3} \lambda^{-2} \iint_{\mathcal{O} \times(0, T)} e^{-2 s \sigma_{1}}\left(\xi_{1}\right)^{-3}|\psi|^{2} d x d t .
$$

Putting together (21) and (20), we obtain

$$
\begin{aligned}
& I_{0}^{1}\left(\gamma^{1}\right)+I_{0}^{2}\left(\gamma^{2}\right)+s^{-3} \lambda^{-2} \iint_{Q} e^{-2 s \sigma_{1}}\left(\xi_{1}\right)^{-3}|\psi|^{2} d x d t \leqslant C\left(\lambda \iint_{\omega_{1} \times(0, T)} e^{-2 s \sigma_{1}}\left|\gamma^{1}\right|^{2} d x d t\right. \\
& \quad+s^{-6} \lambda^{-6} \iint_{\mathcal{O}_{1, d} \times(0, T)} e^{-2 s \sigma_{1}}\left(\xi_{1}\right)^{-6}\left|\gamma^{1}\right|^{2} d x d t+\lambda \iint_{\omega_{2} \times(0, T)} e^{-2 s \sigma_{2}}\left|\gamma^{2}\right|^{2} d x d t \\
& \quad+s^{-6} \lambda^{-6} \iint_{\mathcal{O}_{2, d} \times(0, T)} e^{-2 s \sigma_{1}}\left(\xi_{1}\right)^{-6}\left|\theta_{3} \gamma^{2}\right|^{2} d x d t+s^{-3} \lambda^{-3} \iint_{\mathcal{O}_{1} \times(0, T)} e^{-2 s \sigma_{1}}\left(\xi_{1}\right)^{-3}|\psi|^{2} d x d t \\
& \left.\quad+s^{-3} \lambda^{-3} \iint_{\mathcal{O}_{2} \times(0, T)} e^{-2 s \sigma_{2}}\left(\xi_{2}\right)^{-3}|\psi|^{2} d x d t+s^{-3} \lambda^{-2} \iint_{\mathcal{O} \times(0, T)} e^{-2 s \sigma_{1}}\left(\xi_{1}\right)^{-3}|\psi|^{2} d x d t\right) .
\end{aligned}
$$

Observe that the second and the fifth terms in the right hand side of (22) can be absorbed by the left hand side by taking $s \geq C T^{2}$ and $\lambda \geq C$ :

$$
\begin{aligned}
& s^{-6} \lambda^{-6} \iint_{\mathcal{O}_{1, d} \times(0, T)} e^{-2 s \sigma_{1}}\left(\xi_{1}\right)^{-6}\left|\gamma^{1}\right|^{2} d x d t+s^{-3} \lambda^{-3} \iint_{\mathcal{O}_{1} \times(0, T)} e^{-2 s \sigma_{1}}\left(\xi_{1}\right)^{-3}|\psi|^{2} d x d t \\
& \quad \leqslant \varepsilon\left(I_{0}^{1}\left(\gamma^{1}\right)+s^{-3} \lambda^{-2} \iint_{Q} e^{-2 s \sigma_{1}}\left(\xi_{1}\right)^{-3}|\psi|^{2} d x d t\right) .
\end{aligned}
$$

The same happens to the fourth term in the right hand side of (22), since $\eta_{1}=\eta_{2}$ (so $\sigma_{1}=\sigma_{2}$ and $\xi_{1}=\xi_{2}$ ) in $\operatorname{Supp}\left(\theta_{3}\right) \cap \mathcal{O}_{2, d}$ :

$$
s^{-6} \lambda^{-6} \iint_{\mathcal{O}_{2, d} \times(0, T)} e^{-2 s \sigma_{1}}\left(\xi_{1}\right)^{-6}\left|\theta_{3} \gamma^{2}\right|^{2} d x d t=s^{-6} \lambda^{-6} \iint_{\mathcal{O}_{2, d} \times(0, T)} e^{-2 s \sigma_{2}}\left(\xi_{2}\right)^{-6}\left|\theta_{3} \gamma^{2}\right|^{2} d x d t \leqslant \varepsilon I_{0}^{2}\left(\gamma^{2}\right) .
$$

Using again that $\eta_{1}=\eta_{2}$ outside $\mathcal{O}$, we can estimate the sixth term in the right hand side of (22) for $\lambda \geq C$ as follows:

$$
\begin{aligned}
& s^{-3} \lambda^{-3} \iint_{\mathcal{O}_{2} \times(0, T)} e^{-2 s \sigma_{2}}\left(\xi_{2}\right)^{-3}|\psi|^{2} d x d t=s^{-3} \lambda^{-3} \iint_{\left(\mathcal{O} \cap \mathcal{O}_{2}\right) \times(0, T)} e^{-2 s \sigma_{2}}\left(\xi_{2}\right)^{-3}|\psi|^{2} d x d t \\
& +s^{-3} \lambda^{-3} \iint_{\left(\mathcal{O}_{2} \backslash \mathcal{O}\right) \times(0, T)} e^{-2 s \sigma_{1}}\left(\xi_{1}\right)^{-3}|\psi|^{2} d x d t \leqslant s^{-3} \lambda^{-3} \iint_{\mathcal{O} \times(0, T)} e^{-2 s \sigma_{2}}\left(\xi_{2}\right)^{-3}|\psi|^{2} d x d t \\
& \quad+\varepsilon s^{-3} \lambda^{-2} \iint_{Q} e^{-2 s \sigma_{1}}\left(\xi_{1}\right)^{-3}|\psi|^{2} d x d t .
\end{aligned}
$$


Plugging the estimates (23)-(25) in (22), we obtain:

$$
\begin{aligned}
& I_{0}^{1}\left(\gamma^{1}\right)+I_{0}^{2}\left(\gamma^{2}\right)+s^{-3} \lambda^{-2} \iint_{Q} e^{-2 s \sigma_{1}}\left(\xi_{1}\right)^{-3}|\psi|^{2} d x d t \leqslant C\left(\lambda \iint_{\omega_{1} \times(0, T)} e^{-2 s \sigma_{1}}\left|\gamma^{1}\right|^{2} d x d t\right. \\
& \left.\quad+\lambda \iint_{\omega_{2} \times(0, T)} e^{-2 s \sigma_{2}}\left|\gamma^{2}\right|^{2} d x d t+s^{-3} \lambda^{-2} \iint_{\mathcal{O} \times(0, T)}\left(e^{-2 s \sigma_{1}}\left(\xi_{1}\right)^{-3}+e^{-2 s \sigma_{2}}\left(\xi_{2}\right)^{-3}\right)|\psi|^{2} d x d t\right) .
\end{aligned}
$$

Now, we are going to estimate the first and the second term on the right hand side of (26). For the first one, let $\widetilde{\omega}_{1}$ be an open set satisfying $\omega_{1} \subset \subset \widetilde{\omega}_{1} \subset \subset \mathcal{O}_{1, d} \cap \widetilde{\mathcal{O}}, \widetilde{\omega}_{1} \cap \mathcal{O}_{2, d}=\emptyset$ and let $\theta_{1} \in C_{c}^{2}\left(\widetilde{\omega}_{1}\right)$ be such that $\theta_{1}(x)=1$ for $x \in \omega_{1}$ and $0 \leqslant \theta_{1} \leqslant 1$. Using the PDE satisfied by $\psi$, integrating by parts in time and in space and using the PDE satisfied by $\gamma_{1}$ (see (11)), we deduce

$$
\begin{aligned}
& \lambda \iint_{\omega_{1} \times(0, T)} e^{-2 s \sigma_{1}}\left|\gamma^{1}\right|^{2} d x d t \leqslant \frac{\lambda}{\alpha_{1}} \iint_{\widetilde{\omega}_{1} \times(0, T)} \theta_{1} e^{-2 s \sigma_{1}} \gamma^{1}\left(-\psi_{t}-\Delta \psi+a \psi\right) d x d t \\
& \quad=\frac{\lambda}{\alpha_{1}} \iint_{\widetilde{\omega}_{1} \times(0, T)}\left[\left(\partial_{t}\left(\theta_{1} e^{-2 s \sigma_{1}}\right)-\Delta\left(\theta_{1} e^{-2 s \sigma_{1}}\right)\right) \gamma^{1}-2 \nabla\left(\theta_{1} e^{-2 s \sigma_{1}}\right) \cdot \nabla \gamma^{1}\right] \psi d x d t \\
& \quad-\frac{\lambda}{\alpha_{1} \mu} \iint_{\widetilde{\omega}_{1} \times(0, T)} \theta_{1} e^{-2 s \sigma_{1}}|\psi|^{2} 1_{\mathcal{O}_{1}} d x d t .
\end{aligned}
$$

Using that

$$
\left|\partial_{t}\left(e^{-2 s \sigma_{1}}\right)\right|+\left|\Delta\left(\theta_{1} e^{-2 s \sigma_{1}}\right)\right| \leqslant C s^{2} \lambda^{2}\left(\xi_{1}\right)^{2} e^{-2 s \sigma_{1}} \quad \text { and } \quad\left|\nabla\left(\theta_{1} e^{-2 s \sigma_{1}}\right)\right| \leqslant C s \lambda \xi_{1} e^{-2 s \sigma_{1}},
$$

for $s \geq C\left(T+T^{2}\right)$ and $\lambda \geq C$, we obtain

$$
\begin{aligned}
& \lambda \iint_{\omega_{1} \times(0, T)} e^{-2 s \sigma_{1}}\left|\gamma^{1}\right|^{2} d x d t \leqslant C s \lambda^{2} \iint_{\widetilde{\omega}_{1} \times(0, T)} \xi_{1} e^{-2 s \sigma_{1}}\left(s \lambda \xi_{1}\left|\gamma^{1}\right|+\left|\nabla \gamma^{1}\right|\right)|\psi| d x d t \\
& \quad \leqslant \varepsilon I_{0}^{1}\left(\gamma^{1}\right)+C s^{4} \lambda^{5} \iint_{\widetilde{\omega}_{1 \times(0, T)}}\left(\xi_{1}\right)^{4} e^{-2 s \sigma_{1}}|\psi|^{2} d x d t .
\end{aligned}
$$

The same computations can be performed in order to estimate the second term in the right hand side of (26). Thus

$$
\lambda \iint_{\omega_{2} \times(0, T)} e^{-2 s \sigma_{2}}\left|\gamma^{2}\right|^{2} d x d t \leqslant \varepsilon I_{0}^{2}\left(\gamma^{2}\right)+C s^{4} \lambda^{5} \iint_{\widetilde{\omega}_{2} \times(0, T)}\left(\xi_{2}\right)^{4} e^{-2 s \sigma_{2}}|\psi|^{2} d x d t,
$$

where $\widetilde{\omega}_{2}$ is an open set satisfying $\omega_{2} \subset \subset \widetilde{\omega}_{2} \subset \subset \mathcal{O}_{2, d} \cap \widetilde{\mathcal{O}}$ and $\widetilde{\omega}_{2} \cap \mathcal{O}_{1, d}=\emptyset$.

In view of (28) and (29) and coming back to (26), we deduce the desired inequality (18).

- Proof of (ii). In the proof of (i), in order to absorb the first and second terms on the right hand side of (26), the assumption (15) was crucial. However, it is clear that (15) does not cover all the cases where (8) holds (see, for instance, Fig. 3 in Remark 2). Thus, in order to treat this situation, we will proceed in a slightly different manner.

We will prove $(19)$ when $\left(i_{0}, j_{0}\right)=(2,1)$, that is to say, in the case of Figure 3 .

Recall that $h=\alpha_{1} \gamma^{1}+\alpha_{2} \gamma^{2}$. Applying Proposition 9 (with $m=0$ and $j=2$ ) to the function $(x, t) \mapsto$ $h(x, T-t)$, one gets

$$
I_{0}^{2}(h) \leqslant C\left(\lambda \iint_{\omega_{2} \times(0, T)} e^{-2 s \sigma_{2}}|h|^{2} d x d t+s^{-3} \lambda^{-3} \sum_{k=1}^{2} \iint_{\mathcal{O}_{k} \times(0, T)} e^{-2 s \sigma_{2}}\left(\xi_{2}\right)^{-3}|\psi|^{2} d x d t\right) .
$$


Summing up (20) for $i=1,(21)$ and (30), we obtain

$$
\begin{aligned}
& I_{0}^{1}\left(\gamma^{1}\right)+I_{0}^{2}(h)+s^{-3} \lambda^{-2} \iint_{Q} e^{-2 s \sigma_{1}}\left(\xi_{1}\right)^{-3}|\psi|^{2} d x d t \leqslant C\left(\lambda \iint_{\omega_{1} \times(0, T)} e^{-2 s \sigma_{1}}\left|\gamma^{1}\right|^{2} d x d t\right. \\
& \quad+s^{-6} \lambda^{-6} \iint_{\mathcal{O}_{1, d} \times(0, T)} e^{-2 s \sigma_{1}}\left(\xi_{1}\right)^{-6}\left|\gamma^{1}\right|^{2} d x d t+\lambda \iint_{\omega_{2} \times(0, T)} e^{-2 s \sigma_{2}}|h|^{2} d x d t \\
& \quad+s^{-6} \lambda^{-6} \iint_{\mathcal{O}_{2, d} \times(0, T)} e^{-2 s \sigma_{1}}\left(\xi_{1}\right)^{-6}\left|\theta_{3} \gamma^{2}\right|^{2} d x d t+s^{-3} \lambda^{-3} \iint_{\mathcal{O}_{1} \times(0, T)} e^{-2 s \sigma_{1}}\left(\xi_{1}\right)^{-3}|\psi|^{2} d x d t \\
& \left.\quad+s^{-3} \lambda^{-3} \sum_{k=1}^{2} \iint_{\mathcal{O}_{k} \times(0, T)} e^{-2 s \sigma_{2}}\left(\xi_{2}\right)^{-3}|\psi|^{2} d x d t+s^{-3} \lambda^{-2} \iint_{\mathcal{O} \times(0, T)} e^{-2 s \sigma_{1}}\left(\xi_{1}\right)^{-3}|\psi|^{2} d x d t\right) .
\end{aligned}
$$

Note that the first term in the right hand side of (31) can be estimated as in (27). For the second and fifth terms, we can proceed as in (23). Using the fact that $\eta_{1}=\eta_{2}$ in $\operatorname{Supp}\left(\theta_{3}\right) \cap \mathcal{O}_{2, d}$, we also see that the fourth term can be estimated as follows:

$$
\begin{aligned}
s^{-6} \lambda^{-6} \iint_{\mathcal{O}_{2, d} \times(0, T)} e^{-2 s \sigma_{1}}\left(\xi_{1}\right)^{-6}\left|\theta_{3} \gamma^{2}\right|^{2} d x d t & \leqslant s^{-6} \lambda^{-6} \iint_{\mathcal{O}_{2, d} \times(0, T)} e^{-2 s \sigma_{1}}\left(\xi_{1}\right)^{-6}\left|\theta_{3} h\right|^{2} d x d t \\
& +s^{-6} \lambda^{-6} \iint_{\mathcal{O}_{2, d} \times(0, T)} e^{-2 s \sigma_{1}}\left(\xi_{1}\right)^{-6}\left|\theta_{3} \gamma^{1}\right|^{2} d x d t \\
& \leqslant \epsilon\left(I_{0}^{2}(h)+I_{0}^{1}\left(\gamma^{1}\right)\right) .
\end{aligned}
$$

For the sixth term, proceeding as in (25), one obtains

$$
\begin{aligned}
s^{-3} \lambda^{-3} \sum_{k=1}^{2} \iint_{\mathcal{O}_{k} \times(0, T)} e^{-2 s \sigma_{2}}\left(\xi_{2}\right)^{-3}|\psi|^{2} d x d t & \leqslant s^{-3} \lambda^{-3} \iint_{\mathcal{O} \times(0, T)} e^{-2 s \sigma_{2}}\left(\xi_{2}\right)^{-3}|\psi|^{2} d x d t \\
& +\varepsilon s^{-3} \lambda^{-2} \iint_{Q} e^{-2 s \sigma_{1}}\left(\xi_{1}\right)^{-3}|\psi|^{2} d x d t .
\end{aligned}
$$

For the third term, let $\widetilde{\omega}_{2}$ be an open set satisfying $\omega_{2} \subset \subset \widetilde{\omega}_{2} \subset \subset \mathcal{O}_{2, d} \cap \widetilde{\mathcal{O}}$ and let $\theta_{2} \in C_{c}^{2}\left(\widetilde{\omega}_{2}\right)$ be such that $\theta_{2}(x)=1$ for $x \in \omega_{2}$ and $0 \leqslant \theta_{2} \leqslant 1$. Using that $-\psi_{t}-\Delta \psi+a \psi=h$ in $\widetilde{\omega}_{2} \times(0, T)$, one has

$$
\begin{aligned}
& \lambda \iint_{\omega_{2} \times(0, T)} e^{-2 s \sigma_{2}}|h|^{2} d x d t \leqslant \lambda \iint_{\widetilde{\omega}_{2} \times(0, T)} \theta_{2} e^{-2 s \sigma_{2}} h\left(-\psi_{t}-\Delta \psi+a \psi\right) d x d t \\
& \quad=\lambda \iint_{\widetilde{\omega}_{2} \times(0, T)}\left[\left(\partial_{t}\left(\theta_{2} e^{-2 s \sigma_{2}}\right)-\Delta\left(\theta_{2} e^{-2 s \sigma_{2}}\right)\right) h-2 \nabla\left(\theta_{2} e^{-2 s \sigma_{2}}\right) \cdot \nabla h\right] \psi d x d t \\
& \quad-\lambda \sum_{k=1}^{2} \frac{1}{\mu} \iint_{\widetilde{\omega}_{2} \times(0, T)} \theta_{2} e^{-2 s \sigma_{2}}|\psi|^{2} 1_{\mathcal{O}_{k}} d x d t .
\end{aligned}
$$

Using that

$$
\left|\partial_{t}\left(e^{-2 s \sigma_{2}}\right)\right|+\left|\Delta\left(\theta_{2} e^{-2 s \sigma_{2}}\right)\right| \leqslant C s^{2} \lambda^{2}\left(\xi_{2}\right)^{2} e^{-2 s \sigma_{2}} \quad \text { and } \quad\left|\nabla\left(\theta_{2} e^{-2 s \sigma_{2}}\right)\right| \leqslant C s \lambda \xi_{2} e^{-2 s \sigma_{2}},
$$

for $s \geq C\left(T+T^{2}\right)$ and $\lambda \geq C$, we obtain from (34) that

$$
\begin{aligned}
\lambda \iint_{\omega_{2} \times(0, T)} e^{-2 s \sigma_{2}}|h|^{2} d x d t & \leqslant C s \lambda^{2} \iint_{\widetilde{\omega}_{2} \times(0, T)} \xi_{2} e^{-2 s \sigma_{2}}\left(s \lambda \xi_{2}|h|+|\nabla h|\right)|\psi| d x d t \\
& \leqslant \varepsilon I_{0}^{2}(h)+C s^{4} \lambda^{5} \iint_{\widetilde{\omega}_{2} \times(0, T)}\left(\xi_{2}\right)^{4} e^{-2 s \sigma_{2}}|\psi|^{2} d x d t .
\end{aligned}
$$


Combining (23), (27), (32), (33), and (35) we deduce the following estimate:

$$
\begin{aligned}
& I_{0}^{1}\left(\gamma^{1}\right)+I_{0}^{2}(h)+s^{-3} \lambda^{-2} \iint_{Q} e^{-2 s \sigma_{1}}\left(\xi_{1}\right)^{-3}|\psi|^{2} d x d t \\
& \quad \leqslant C s^{4} \lambda^{5} \iint_{\mathcal{O} \times(0, T)}\left(e^{-2 s \sigma_{1}}\left(\xi_{1}\right)^{4}+e^{-2 s \sigma_{2}}\left(\xi_{2}\right)^{4}\right)|\psi|^{2} d x d t .
\end{aligned}
$$

This ends the proof.

Remark 13. A somewhat easier proof of Proposition 11 can be deduced using the fact that $\left\|\eta_{1}\right\|_{\infty}=\left\|\eta_{2}\right\|_{\infty}$ (see Remark 7), but we have preferred to provide an argument which applies also in the more general case, with $\left\|\eta_{1}\right\|_{\infty} \neq\left\|\eta_{2}\right\|_{\infty}$.

\section{Observability and null controllability}

As already announced, to achieve the proof of Theorem 1, we will apply a standard controllabilityobservability argument. Thus, it will be sufficient to prove (12).

We will need the following:

Lemma 14. There exists $\mu_{00}$ such that, for any $\mu \geq \mu_{00}$ and any $t_{1}, t_{2}$ with $0 \leqslant t_{1} \leqslant t_{2} \leqslant T$, the solution $\left(\psi, \gamma^{i}\right)$ to (11) satisfies

$$
\left\|\psi\left(\cdot, t_{1}\right)\right\|^{2} \leqslant C\left\|\psi\left(\cdot, t_{2}\right)\right\|^{2}
$$

where $C>0$ only depends on $\Omega, \mathcal{O}, T, \mathcal{O}_{i}, \mathcal{O}_{i, d}, \alpha_{i}$ and $\|a\|_{L^{\infty}(Q)}$.

Proof. Multiplying $(11)_{2}$ by $\gamma^{i}$ and integrating in $(0, t) \times \Omega$, it follows that

$$
\left\|\gamma^{i}(\cdot, t)\right\|^{2}+2 \int_{0}^{t}\left\|\nabla \gamma^{i}(\cdot, s)\right\|^{2} d s \leqslant 2\left(\|a\|_{\infty}+1\right) \int_{0}^{t}\left\|\gamma^{i}(\cdot, s)\right\|^{2} d s+\frac{1}{2 \mu^{2}} \int_{0}^{t}\|\psi(\cdot, s)\|^{2} d s .
$$

From Gronwall's Lemma, we have

$$
\left\|\gamma^{i}(\cdot, t)\right\|^{2} \leqslant \frac{C}{\mu^{2}} \int_{0}^{t}\|\psi(\cdot, s)\|^{2} d s
$$

and, consequently,

$$
\int_{t}^{t^{\prime}}\left\|\gamma^{i}(\cdot, s)\right\|^{2} d s \leqslant \frac{C}{\mu^{2}} \int_{0}^{t^{\prime}}\|\psi(\cdot, s)\|^{2} d s, \quad \forall t^{\prime} \in[t, T]
$$

Now, multiplying $(11)_{1}$ by $\psi$ and integrating in $\left(t, t^{\prime}\right) \times \Omega \subset Q$, one gets

$$
\|\psi(\cdot, t)\|^{2}+2 \int_{t}^{t^{\prime}}\|\nabla \psi(\cdot, s)\|^{2} d s \leqslant\left\|\psi\left(\cdot, t^{\prime}\right)\right\|^{2}+2\left(\|a\|_{\infty}+1\right) \int_{t}^{t^{\prime}}\|\psi(\cdot, s)\|^{2} d s+\sum_{i=1}^{2} \frac{\left|\alpha_{i}\right|^{2}}{2} \int_{t}^{t^{\prime}}\left\|\gamma^{i}(\cdot, s)\right\|^{2} d s,
$$

which, used in combination with (37), gives

$$
\|\psi(\cdot, t)\|^{2}+2 \int_{t}^{t^{\prime}}\|\nabla \psi(\cdot, s)\|^{2} d s \leqslant\left\|\psi\left(\cdot, t^{\prime}\right)\right\|^{2}+\frac{C}{\mu^{2}} \int_{0}^{t^{\prime}}\|\psi(\cdot, s)\|^{2} d s+2\left(\|a\|_{\infty}+1\right) \int_{t}^{t^{\prime}}\|\psi(\cdot, s)\|^{2} d s .
$$

In view again of Gronwall's Lemma, we see that

$$
\|\psi(\cdot, t)\|^{2} \leqslant C\left\|\psi\left(\cdot, t^{\prime}\right)\right\|^{2}+\frac{C}{\mu^{2}} \int_{0}^{t^{\prime}}\|\psi(\cdot, s)\|^{2} d s .
$$

and, therefore, integrating this inequality from 0 to $t^{\prime}$ and taking $\mu$ large enough, we can guarantee that

$$
\int_{0}^{t^{\prime}}\|\psi(\cdot, s)\|^{2} d s \leqslant C\left\|\psi\left(\cdot, t^{\prime}\right)\right\|^{2} .
$$

From this estimate and (38), we deduce (36). 
We are now prepared to present the proof of Theorem 3.

Proof of Theorem 3. Using the inequalities

$$
e^{-2 s \sigma_{i}} t^{-3}(T-t)^{-3} \geq e^{-C(1+1 / T)} \frac{1}{T^{6}} \quad \text { in } \quad \Omega \times(T / 4,3 T / 4)
$$

and

$$
e^{-2 s \sigma_{i}} t^{-3}(T-t)^{-3} \leqslant e^{-C(1+1 / T)} \frac{1}{T^{6}} \quad \text { in } \quad Q,
$$

we get from (18) or (19) that

$$
\iint_{\Omega \times(T / 4,3 T / 4)}|\psi|^{2} d x d t \leqslant C \iint_{\mathcal{O} \times(0, T)}|\psi|^{2} d x d t .
$$

On the other hand, according to Lemma 14, one has

$$
\|\psi(\cdot, 0)\|^{2} \leqslant C\|\psi(\cdot, t)\|^{2} \quad \forall t \in(T / 4,3 T / 4) .
$$

Combining (39) and (40), we deduce that

$$
\|\psi(\cdot, 0)\|^{2} \leqslant C \iint_{\mathcal{O} \times(0, T)}|\psi|^{2} d x d t
$$

In order to obtain (12), we have to add global weighted integrals of $\gamma^{i}$ in the left hand side of (41). We will again consider separately two possibilities:

- Case 1: Condition (i) of Proposition 11 is satisfied.

In this case, the observability estimate (12) follows directly from (41).

In fact, let us consider the following weight functions

$$
\ell(t):= \begin{cases}T^{2} / 4 & \text { for } \quad 0 \leqslant t \leqslant T / 2 \\ t(T-t) & \text { for } \quad T / 2 \leqslant t \leqslant T\end{cases}
$$

and

$$
\bar{\sigma}_{i}(x, t):=\frac{e^{4 \lambda\left\|\eta_{i}\right\|_{\infty}}-e^{\lambda\left(2\left\|\eta_{i}\right\|_{\infty}+\eta_{i}(x)\right)}}{\ell(t)}, \quad \bar{\xi}_{i}(x, t):=\frac{e^{\lambda\left(2\left\|\eta_{i}\right\|_{\infty}+\eta_{i}(x)\right)}}{\ell(t)} .
$$

One can directly see from the energy inequality (36) and the Carleman inequality (18) that there exists $C>0$ such that

$$
s^{-3} \lambda^{-2} \iint_{Q} e^{-2 s \bar{\sigma}_{1}}\left(\bar{\xi}_{1}\right)^{-3}|\psi|^{2} d x d t \leqslant C s^{4} \lambda^{5} \iint_{\mathcal{O} \times(0, T)}\left(e^{-2 s \bar{\sigma}_{1}}\left(\bar{\xi}_{1}\right)^{4}+e^{-2 s \bar{\sigma}_{2}}\left(\bar{\xi}_{2}\right)^{4}\right)|\psi|^{2} d x d t
$$

(here, we use that $\mu>0$ is large enough).

Let $\hat{\rho}=\hat{\rho}(t)$ be a positive nondecreasing $C^{1}$ function which blows up at $t=T$. From the PDE satisfied by $\gamma^{i}$ in (11), we readily see that

$$
\begin{aligned}
& \frac{1}{2} \frac{d}{d t} \int_{\Omega} \hat{\rho}^{-2}\left|\gamma^{i}\right|^{2} d x+\int_{\Omega} \hat{\rho}^{-2}\left|\nabla \gamma^{i}\right|^{2} d x \\
& \quad=-\frac{1}{\mu} \int_{\mathcal{O}_{i}} \hat{\rho}^{-2} \psi \gamma^{i} d x-\int_{\Omega} \hat{\rho}^{-3} \hat{\rho}_{t}\left|\gamma^{i}\right|^{2} d x-\int_{\Omega} \hat{\rho}^{-2} a\left|\gamma^{i}\right|^{2} d x \\
& \quad \leqslant \frac{1}{\mu^{2}} \int_{\mathcal{O}_{i}} \hat{\rho}^{-2}|\psi|^{2} d x+\left(1+\|a\|_{\infty}\right) \int_{\Omega} \hat{\rho}^{-2}\left|\gamma^{i}\right|^{2} d x
\end{aligned}
$$


and, using Gronwall's Lemma and the fact that $\gamma^{i}(x, 0) \equiv 0$, it follows that

$$
\left(\int_{\Omega} \hat{\rho}^{-2}\left|\gamma^{i}\right|^{2} d x\right)(\tau) \leqslant C \iint_{\mathcal{O}_{i} \times(0, T)} \hat{\rho}^{-2}|\psi|^{2} d x d t, \quad \forall \tau \in[0, T] .
$$

Let $\bar{\sigma}_{1}^{*}(t)=\max _{x \in \bar{\Omega}} \bar{\sigma}_{1}(x, t)$ and choose $\hat{\rho}(t)=e^{s \bar{\sigma}_{1}^{*}(t)}$. Note that the choice of $\hat{\rho}$ is determined by the Carleman weight $e^{s \sigma}$, that depends on $\Omega, \mathcal{O}, T$ and $\|a\|_{L^{\infty}(Q)}$; but $\hat{\rho}$ can be chosen independent of the $\mathcal{O}$, $\mathcal{O}_{i, d}, \alpha_{i}$ and $\mu$. Since

$$
\iint_{\mathcal{O}_{i} \times(0, T)} \hat{\rho}^{-2}|\psi|^{2} d x d t \leqslant C \iint_{Q}\left(\bar{\xi}_{1}\right)^{-3} e^{-2 s \bar{\sigma}_{1}}|\psi|^{2} d x d t
$$

in view of (42) and (43), we find that

$$
\sum_{i=1}^{2} \iint_{Q} \hat{\rho}^{-2}\left|\gamma^{i}\right|^{2} d x d t \leqslant C \iint_{\mathcal{O} \times(0, T)}\left(e^{-2 s \bar{\sigma}_{1}}\left(\bar{\xi}_{1}\right)^{4}+e^{-2 s \bar{\sigma}_{2}}\left(\bar{\xi}_{2}\right)^{4}\right)|\psi|^{2} d x d t .
$$

Combining this last inequality and (41), we deduce the observability estimate (12).

- Case 2: Condition (ii) of Proposition 11 is satisfied.

The proof in this case is exactly the same as in the first case since in the proof of the first case we only used the third term in the left-hand side of (18), but we also have this term in the left-hand side of (19).

\section{Appendix: Proof of Lemma 5}

Let us first consider a function $f \in C^{2}(\bar{\Omega})$ such that $f>0$ in $\Omega, f=0$ on $\partial \Omega$ and the set

$$
A_{f}:=\{x \in \bar{\Omega}: \nabla f(x)=0\}
$$

is finite and does not intersect $\partial \Omega$. The construction of $f$ can be achieved following classical arguments of Morse theory (see [8, pp. 20-21] for more details).

Let us indicate how the functions $\eta_{1}$ and $\eta_{2}$ can be constructed:

Construction of $\eta_{1}$ : Let us set $A_{f}:=\left\{a_{m}: 1 \leqslant i \leqslant k\right\}$. Then, from the connectedness of $\Omega$, one can construct $k$ bijective paths $\zeta_{m} \in C^{\infty}([0,1] ; \Omega)$ such that $\zeta_{m}(0)=a_{m}, \zeta_{m}(1):=b_{m} \in \omega_{1}$ and

$$
\zeta_{m}([0,1]) \cap \zeta_{r}([0,1])=\emptyset \text { for } m \neq r .
$$

Now, let $G \in C^{\infty}\left(\mathbb{R}^{n} ; \mathbb{R}^{n}\right)$ be such that $\operatorname{Supp}(G) \subset \Omega$ and

$$
G\left(\zeta_{m}(t)\right)=\zeta_{m}^{\prime}(t) \quad \forall t \in[0,1], \forall m \in\{1, \ldots, k\} .
$$

Note that the function $G$ can be first defined in the compact set $\zeta_{m}([0,1])$ as above and then extended as a $C^{\infty}$ function to a neighborhood of $\zeta_{m}([0,1])$.

Let $\Theta=\Theta(x, t)$ be the flow associated to $G$, that is to say, the solution to

$$
\begin{cases}\frac{\partial \Theta}{\partial t}(x, t)=G(\Theta(x, t)) & \text { for }(x, t) \in \mathbb{R}^{n} \times \mathbb{R}, \\ \Theta(x, 0)=x & \text { for } x \in \mathbb{R}^{n} .\end{cases}
$$

From (44) and the uniqueness of solution of the Cauchy problem for an ODE, we necessarily have that $\Theta\left(a_{m}, t\right)=\zeta_{m}(t)$ for all $t \in[0,1]$, whence $\Theta\left(a_{m}, 1\right)=b_{m} \in \omega_{1}$ for all $m$.

We remark that, for all $t \in \mathbb{R}$, the function $\Theta(t, \cdot)$ satisfies the following properties:

- It is a diffeomorphism on $\mathbb{R}^{n}$ (with inverse $\Theta(\cdot,-t)$ );

- It maps $\Omega$ into itself (since $\operatorname{Supp}(G) \subset \Omega$ ); 
- It coincides with the identity map on a neighborhood of $\partial \Omega$.

Then the function $\eta_{1}(x):=f(\Theta(x,-1))$ (defined in the whole $\bar{\Omega}$ ) satisfies $\eta_{1}>0$ in $\Omega, \eta_{1}=0$ on $\partial \Omega$, $\eta_{1} \in C^{2}(\bar{\Omega})$ and

$$
\nabla \eta_{1}(x)=0 \Leftrightarrow \exists m \in\{1, \ldots, k\} \text { such that } x=b_{m} .
$$

Construction of $\eta_{2}$ : Let us now consider $k$ bijective paths $\delta_{m} \in C^{\infty}([0,1] ; \widetilde{\mathcal{O}})$ such that $\delta_{m}(0)=b_{m}$, $\delta_{m}(1):=c_{m} \in \omega_{2}$ and

$$
\delta_{m}([0,1]) \cap \delta_{j}([0,1])=\emptyset \text { for } i \neq j .
$$

Let $H \in C^{\infty}\left(\mathbb{R}^{n} ; \mathbb{R}^{n}\right)$ be such that $\operatorname{Supp}(H) \subset \widetilde{\mathcal{O}}$ and

$$
H\left(\delta_{m}(t)\right)=\delta_{m}^{\prime}(t), \quad \forall t \in[0,1], \forall m \in\{1, \ldots, k\} .
$$

Next, let $\Pi=\Pi(x, t)$ be the flow associated to $H$, that is,

$$
\begin{cases}\frac{\partial \Pi}{\partial t}(x, t)=H(\Pi(x, t)) & \text { for }(x, t) \in \mathbb{R}^{n} \times \mathbb{R}, \\ \Pi(x, 0)=x & \text { for } x \in \mathbb{R}^{n} .\end{cases}
$$

Then, if we introduce the function

$$
\eta_{2}(x):= \begin{cases}\eta_{1}(x) & \text { if } x \in \bar{\Omega} \backslash \widetilde{\mathcal{O}}, \\ \eta_{1}(\Pi(x,-1)) & \text { if } x \in \overline{\widetilde{\mathcal{O}}}\end{cases}
$$

we see that $\eta_{2}>0$ in $\Omega, \eta_{2}=0$ on $\partial \Omega, \eta_{2} \in C^{2}(\bar{\Omega})$ (observe that $\Pi(-1, \cdot)$ is the identity map in a neighborhood of $\partial \widetilde{\mathcal{O}}$ ) and

$$
\nabla \eta_{2}(x)=0 \Leftrightarrow \exists m \in\{1, \ldots, k\} \text { such that } x=c_{m} .
$$

The proof of Lemma 5 is thus complete.

\section{References}

[1] F.D. Araruna, E. Fernández-Cara, M.C. Santos, Stackelberg-Nash exact controllability for linear and semilinear parabolic equations. ESAIM Control Optim. Calc. Var., 21 (3) (2015), 835-856.

[2] H. BrÉzis, Analyse fonctionnelle, théorie et applications. Dunod, Paris (1999).

[3] J.C. Cox, M. Rubinstein, Ôptions Markets. Prentice-Hall, Englewood Cliffs, NJ (1985).

[4] J.I. Díaz, On the von Neumann problem and the approximate controllability of Stackelberg-Nash strategies for some environmental problems. Rev. R. Acad. Cien., Serie A. Math. 96 (3) (2002), 343-356.

[5] J.I. Díaz, J.-L. Lions, it On the approximate controllability of Stackelberg-Nash strategies. Ocean circulation and pollution control: a mathematical and numerical investigation (Madrid, 1997). 17-27, Springer, Berlin (2004).

[6] E. Fernández-Cara, S. Guerrero, Global Carleman inequalities for parabolic systems and applications to controllability. SIAM J. Control Optimiz. 45 (4) (2006), 1395-1446.

[7] E. Fernández-Cara, S. Guerrero, O.Y. Imanuvilov, J.-P. Puel, Local exact controllability of the Navier-Stokes system. J. Math. Pures Appl. 83 (2004), 1501-1542.

[8] A.V. Fursikov, O.Y. Imanuvilov, Controllability of evolution equations. Lecture Note Series 34, Research Institute of Mathematics, Seoul National University, Seoul (1996). 
[9] A.V. Fursikov, O.Y. Imanuvilov, Exact controllability of the Navier-Stokes and Boussinesq equations. Russian Math. Surveys 54 (3) (1999), 565-618.

[10] M. González-Burgos, S. Guerrero, J.-P. Puel, Local exact controllability to the trajectories of the Boussinesq system via a fictitious control on the divergence equation. Commun. Pure Applied Analysis $\mathbf{8}$ (1) (2009), 311-333.

[11] S. Guerrero, O.Y. Imanuvilov, J.-P. Puel, A result concerning the global approximate controllability of the Navier-Stokes system in dimension 3. J. Math. Pures Appl. 98 (6) (2012), 689-709.

[12] F. Guillén-González, F.P. Marques-Lopes, M.A. Rojas-Medar, On the approximate controllability of Stackelberg-Nash strategies for Stokes equations. Proc. Amer. Math. Soc. 141 (5) (2013), 1759-1773.

[13] O.Y. ImanuviLov, Remarks on exact controllability for the Navier-Stokes equations. ESAIM: Control Optim. Calc. Var. 6 (2001), 39-72.

[14] O.Y. Imanuvilov, M. Yamamoto, Carleman estimates for a parabolic equation in a Sobolev space of negative order and its applications, Lecture Notes in Pure and Appl. Math., vol. 218, Dekker, New York (2001).

[15] J.-L. Lions, Contrôle de Pareto de systèmes distribués. Le cas d'évolution. C.R. Acad. Sc. Paris, série I 302 (11) (1986), 413-417.

[16] J.-L. Lions, Some remarks on Stackelberg's optimization. Mathematical Models and Methods in Applied Sciences 4 (1994) 477-487.

[17] J.F. NAsh, Noncooperative games. Annals of Mathematics 54 (1951), 286-295.

[18] V. Pareto, Cours d'économie politique. Rouge, Laussane, Switzerland (1896).

[19] A.M. Ramos, R. Glowinski, J. Periaux, Nash equilibria for the multiobjective control of linear partial differential equations. J. Optim. Theory Appl. 112 (2002), 457-498.

[20] A.M. Ramos, R. Glowinski, J. Periaux, Pointwise control of the Burgers equation and related Nash equilibria problems: A computational approach. J. Optim. Theory Appl. 112 (2001), 499-516.

[21] S.M. Ross, An introduction to mathematical finance. Options and other topics. Cambridge University Press, Cambridge (1999).

[22] H. Von Stalckelberg, Marktform und gleichgewicht. Springer, Berlin, Germany (1934).

[23] P. Wilmott, S. Howison, J. Dewynne, The mathematics of financial derivatives. Cambridge University Press, New York (1995).

[24] E. ZuAzuA, Exact controllability for the semilinear wave equation. J. Math. Pures Appl. 69 (1) (1990), $1-31$. 